\title{
Describing Network Traffic Using the Index of Variability
}

\author{
Georgios Y. Lazarou \\ Department of Electrical \& Computer Engineering, Mississippi State University \\ glaz@ece.msstate.edu \\ Julie Baca \\ Center for Advanced Vehicular Systems, Mississippi State University \\ baca@cavs.msstate.edu
}

Victor S. Frost and Joseph B. Evans

Department of Electrical Engineering \& Computer Science, University of Kansas

$\{$ frost, evans\}@eecs.ku.edu

\begin{abstract}
Commonly used measures of traffic burstiness do not capture the fluctuation of traffic variability over the entire range of time scales. In this paper, we present a measure of variability, called the Index of Variability $\left(H_{v}(\tau)\right)$, that fully and accurately captures the degree of variability (burstiness) of a typical network traffic process at each time scale and is analytically tractable for many traffic models. As an illustration, we derive the closed-form expression of $H_{v}(\tau)$ for two traditional traffic models and generate a variety of $2 \mathrm{D}$ and 3D Index of Variability curves. These curves demonstrate that the Index of Variability is a mathematically rigorous measure which can be used to fully characterize the complexities of the network traffic variability over all time scales. We then introduce a practical method for estimating the Index of Variability curve from a given traffic trace. Experimental results are presented which demonstrate the robustness of the method applied to the estimation of the Index of Variability curves from 12 NLANR network traffic long traces.
\end{abstract}

\section{INTRODUCTION}

Many empirical studies have shown that Internet traffic exhibits high variability ${ }^{1}$ [2], [3], [4], [5], i.e., traffic is bursty (variable) over a wide range of time scales in sharp contrast to the assumption that traffic burstiness exists only at short time scales while traffic is smooth at large time scales [4]. High variability in traffic has been shown to have a significant impact on network performance [4], [6]. The results from [6], [7], [8], [9] show that knowledge of the traffic characteristics on multiple time scales helps to improve the efficiency of

Part of this paper was presented at MSO'03 [1], (C)IASTED 2003.

${ }^{1}$ Fluctuation of traffic as a function of time. traffic control mechanisms. More importantly, the design and provision of quality-of-service-guarantees over the Internet requires the understanding of traffic characteristics, such as variability.

Since the publication of [4], the popular belief has been that the high variability in traffic is due to the long-range dependence (LRD) property of the traffic processes. In general, a (weakly) stationary discrete-time real-valued stochastic process $Y=\left\{Y_{n}, n=0,1,2, \ldots\right\}$ with mean $\mu=E\left[Y_{n}\right]$ and variance $\sigma^{2}=E\left[\left(Y_{n}-\mu\right)^{2}\right]<\infty$ is long-range dependent if $\sum_{k=1}^{\infty} r(k)=\infty$, where $r(k)$ measures the correlation between samples of $Y$ separated by $k$ units of time. If $\sum_{k=1}^{\infty} r(k)<\infty$, then $Y$ is said to exhibit short-range dependence (SRD).

Common traffic models with LRD are based on selfsimilar processes. In traffic modeling, the term selfsimilarity usually refers to the asymptotically second order self-similar or mono-fractal processes [10]. The definition of asymptotically second order self-similarity is as follow [4]: assume that $Y$ has an autocorrelation function of the form $r(k) \sim k^{-\beta} L(k)$ as $k \rightarrow \infty$, where $0<\beta<1$ and the function $L$ is slowly varying at infinity, i.e., $\lim _{k \rightarrow \infty} \frac{L(k x)}{L(k)}=1 \forall x>0$. For each $m=1,2,3, \ldots$, let $Y^{(m)}=\left\{Y_{n}^{(m)}, n=1,2,3, \ldots\right\}$ denote a new aggregated time series obtained by averaging the original series $Y$ over non-overlapping blocks of size $m$, replacing each block by its sample mean. That is, for each $m=$ $1,2,3, \ldots, Y^{(m)}$ is given by

$$
Y_{n}^{(m)}=\frac{Y_{n m-m+1}+\cdots+Y_{n m}}{m} \quad n \geq 1 .
$$

The new aggregated discrete-time stochastic process $Y^{(m)}$ is also (weakly) stationary with an autocorrelation function $r^{(m)}(k)$; then, $Y$ is called asymptotically second 
order self-similar with self-similar parameter $H=1-\frac{\beta}{2}$ if for all $k$ large enough, $r^{(m)}(k) \rightarrow r(k)$ as $m \rightarrow \infty$, that is, $Y$ is asymptotically second-order self-similar if the corresponding aggregated processes $Y^{(m)}$ become indistinguishable from $Y$ at least with respect to their autocorrelation functions. By definition, asymptotically second order self-similarity implies LRD and vice versa [10].

The parameter $H$ is called the Hurst parameter. For general self-similar processes, it measures the degree of "self-similarity". For random processes suitable for modeling network traffic, the Hurst parameter is basically a measure of the speed of decay of the tail of the autocorrelation function. If $0.5<H<1$, then the process is LRD, and if $0<H \leq 0.5$, then it is SRD. Hence, $H$ is widely used to capture the intensity of long-range dependence of a traffic process: the closer $H$ is to 1 the more long-range dependent the traffic is, and vice versa [10].

Several methods exist for estimating $H$ from a traffic trace. One of the most widely used is the Aggregated Variance method: for successive values of $m$ that are equidistant on a $\log$ scale, the sample variance of $Y^{(m)}$ is plotted versus $m$ on a log-log plot [11][12]. By fitting a least-square line to the points of the plot and then calculating its slope, an estimate of the Hurst parameter is obtained as $\hat{H}=1-\frac{\text { slope }}{2}$.

Another very popular method is based on wavelets [13]. Given a traffic trace $Y_{n}$, the Hurst parameter can be estimated as follows: for each scale $j$, the wavelet energy $\mu_{j}=\frac{1}{N_{j}} \sum_{k=1}^{n_{j}} d^{2}(j, k)$ is plotted versus $j$ on a semi-log plot (i.e., $\log _{2}\left(\mu_{j}\right)$ vs. $j$ ). By fitting a least-square line to the points of the curve region that looks linear and then computing its slope $\alpha, H$ is estimated as $\hat{H}=\frac{\alpha+1}{2}$.

\section{A. Need for a New Measure of Variability}

Commonly used measures of traffic burstiness, such as the peak-to-mean ratio, the coefficient of variation of interarrival times, the indices of dispersion for intervals and counts, and the Hurst parameter, do not capture the fluctuation of variability over different time scales.

It is claimed in [4] that the Hurst parameter is a good measure of variability, and the higher the value of $H$, the burstier the traffic. The popular belief from early studies [6], [14], [15], [16] on the impact of LRD on network performance is that high values of the Hurst parameter are associated with poor queueing performance. However, later studies [8], [17] show examples in which larger values of $H$ are associated with better queueing performance compared to smaller values of $H$. In addition, the results in [9] indicate that the

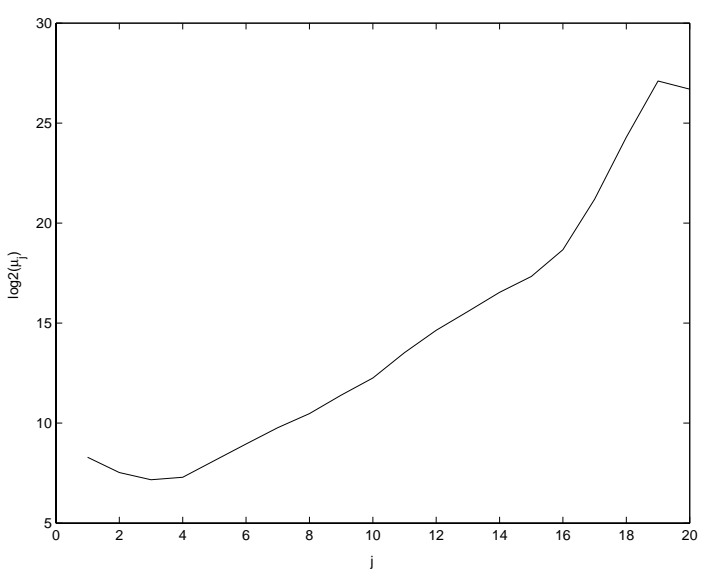

Fig. 1. $\log _{2}\left(\mu_{j}\right)$ versus scale $j$ for the Auckland-IV traffic trace 20010301-310-0 (For information about the trace, see Section IV).

queueing performance depends mostly on the variability over certain time scales rather than on the value of $H$.

Moreover, it is known [7] that different long-range dependent processes with the same value of the Hurst parameter can generate vastly different queueing behavior. Clearly, the single value Hurst parameter does not capture the fluctuation of the degree of traffic burstiness across time scales, regardless of whether the traffic process exhibits LRD or SRD. From the definition presented in the previous section, the Hurst parameter is defined asymptotically (i.e., for large time scales) and hence conveys nothing about the variability of measured traffic over small or medium time scales; unless the traffic is exactly self-similar with known variances. Therefore, the Hurst paremeter is an incomplete descriptor of traffic variability.

For many network traffic processes, the wavelet energy-scale or variance-time plots usually do not tend to straight lines, i.e., see Figure 1. Usually many of these processes have piecewise fractal behavior with varying Hurst parameter over some small ranges of time scales [18]. Such processes are usually referred to as multifractal [19].

Queueing performace greatly depends on traffic irregularities at small time scales which are typically attributed to the complex dynamics of data networks [7], [20]. Multifractal analysis based on the legendre spectrum is often used to study the multiscaling behavior of traffic at small time scales [18], [21], [22], [23]. The process of estimating the legendre spectrum involves higher order sample moments and negative values of moments. It is known [24] that higher order sample moments are not well-behaved and negative values of moments tend to be erratic. In addition, the legendre spectrum is difficult to interpret [25]. 
Hence, there is a need for an intuitively appealing, conceptually simple, and mathematically rigorous measure which can capture the various scaling phenomena that are observed in data networks on both small and large scales [26]. In this paper, we present an alternative measure of variability, called the Index of Variability $\left(H_{v}(\tau)\right)$, that fully and accurately captures the degree of variability of a typical network traffic process at each time scale and is analytically tractable for many traffic models.

The remainder of this paper is organized as follows: Section II presents a rigorous definition of the Index of Variability. Section III presents the derivation of the closed-form expressions of $H_{v}(\tau)$ for two traditional traffic models. A variety of 2D and 3D Index of Variability curves are also presented for illustration. Section IV presents a practical method for estimating the Index of Variability curve from a given traffic trace. Experimental results are also presented to demonstrate the robustness of the method. The paper concludes in Section V.

\section{INDEX OF VARIABILITY FOR PACKET TRAFFIC SEQUENCES}

Let $N(t)$ denote the number of events (packet arrivals) of a stationary point process in the interval $(0, t]$. For each fixed time interval $\tau>0$, an event count sequence $Y=\left\{Y_{n}(\tau), \tau>0, n=1,2, \ldots\right\}$ can be constructed from each point process, where

$$
Y_{n}(\tau)=N[n \tau]-N[(n-1) \tau]
$$

denotes the number of events that have occurred during the $n^{\text {th }}$ time interval of duration $\tau$. Clearly, $Y$ is also (weakly) stationary for all $\tau>0$. In this study, $Y$ represents a network traffic trace where $Y_{n}(\tau)$ denotes the number of packets observed from an arbitrary point in the network during the $n^{\text {th }}$ time interval of duration $\tau$. We refer to $\tau$ as the time scale of the traffic trace, and it represents the length (i.e., $10 \mathrm{~ms}, 1 \mathrm{~s}, 10 \mathrm{~s}$, e.t.c.) of one sample of $Y$.

The expected number of events that have occurred during the interval $(0, t]$ is always: $E[N(t)]=\frac{t}{E[X]}=$ $\lambda t$ where $E[X]$ is the expected interarrival time and $\lambda$ is the mean event (packet) arrival rate. The index of dispersion for counts (IDC) is defined as: $I D C(t) \equiv$ $\frac{\operatorname{Var}[N(t)]}{E[N(t)]}=\frac{\operatorname{Var}[N(t)]}{\lambda t}$. The IDC was defined such that it provides some comparison with the Poisson process, for which $I D C(t)=1 \forall t$. Note that since the point process is stationary, IDC has the same value over any interval of length $t$; thus, $t$ can be viewed as the time scale $\tau$ of the traffic process $Y$ defined in (2). Henceforth $t$ will be used to denote generality and $\tau$ to denote time scales, i.e., the time length of each sample of the packet-count sequence $Y$.

An important feature of IDC is that it is mathematically equivalent to the Aggregated Variance method for estimating the Hurst parameter $H$ of a self-similar process. For a self-similar process, plotting $\log (I D C(m \tau))$ against $\log (m)$ results in an asymptotic straight line with slope $2 H-1$. When $Y$ is a long-range dependent process, the slowly decaying variance property of LRD processes [4] with parameter $0<\beta<1$ is equivalent to an IDC curve $^{2}$ with an asymptotic straight line with slope $1-\beta$, implying $0<$ slope $<1$. When the IDC curve converges to an asymptotic straight line with slope $=0$ for some $\tau<\infty$, then $Y$ is a short-range dependent process. Based on the above property of IDC, we define the following new measure of variability:

Definition 1: For a general stationary traffic process $Y$ as defined by (2) whose $I D C(\tau)$ is continuous and differentiable over $(0, \infty)$, we refer to:

$$
H_{v}(\tau) \equiv \frac{\frac{d(\log (I D C(\tau)))}{d(\log (\tau))}+1}{2}
$$

as the Index of Variability of $Y$ for the time scale $\tau$, where $\frac{d(\log (I D C(\tau)))}{d(\log (\tau))}$ is the local slope of the IDC curve at each $\tau$ when plotted in log-log coordinates.

Note that the index of variability is so defined in order for a long-range dependent (asymptotically or secondorder self-similar) process $H_{v}(\tau)=H \in(0.5,1)$ for all $\tau \geq \tau_{o}>0$. The value of $\tau_{o}$ depends on the particular process. If the process is exactly self-similar, then $H_{v}(\tau)=H \in(0.5,1)$ for all $\tau>0$, that is, if $\log (\operatorname{IDC}(\tau))$ is linear with respect to $\log (\tau))$, then $H_{v}(\tau)$ reduces to $H$. The Index of Variability can be viewed as the Hurst parameter defined at each time scale.

In general ${ }^{3}$, the process $Y$ exhibits significant variability for those time scales $\tau$ such that $0.5<H_{v}(\tau)<$ 1. When $\frac{d(\log (I D C(\tau)))}{d(\log (\tau))} \rightarrow 1$, then $H_{v}(\tau) \rightarrow 1$, implying very high variability. A plot of $H_{v}(\tau)$ versus $\tau$ would depict the behavior of the traffic process $Y$ in terms of variability (burstiness) at each time scale $\tau$ (= $10 \mathrm{~ms}, 100 \mathrm{~ms}, 1 \mathrm{~s}, \ldots)$.

Expanding the local slope of the IDC curve at each time scale, we obtain:

$$
\begin{aligned}
\frac{d(\log (I D C(\tau)))}{d(\log (\tau))} & =\frac{\tau}{\operatorname{IDC}(\tau)} \frac{d(I D C(\tau))}{d \tau} \\
& =\frac{\tau}{\operatorname{Var}[N(\tau)]} \frac{d(\operatorname{Var}[N(\tau)])}{d \tau}-1 .(4)
\end{aligned}
$$

\footnotetext{
${ }^{2}$ In log-log coordinates.

${ }^{3}$ The generality here is confined for those processes that are suitable in modeling network packet traffic.
} 
Using the above in (3), we obtain a more convenient form of the Index of Variability:

$$
\begin{aligned}
H_{v}(\tau) & =0.5 \tau\left(\frac{\frac{d \operatorname{Var}[N(\tau)]}{d \tau}}{\operatorname{Var}[N(\tau)]}\right) \\
& =\frac{1}{2}\left\{1+\tau\left(\frac{\frac{d(I D C(\tau))}{d \tau}}{I D C(\tau)}\right)\right\}
\end{aligned}
$$

In addition, setting $\tau=m T$, where $T>0$ and $m=$ $1,2, \cdots$, and using the relation $\operatorname{Var}\left[Y^{(m)}\right]=\frac{\operatorname{Var}[N(m T)]}{m^{2}}$, we can express the index of variability function in terms of $\operatorname{Var}\left[Y^{(m)}\right]$ versus $m$ :

$$
H_{v}(m T)=0.5 m \frac{\frac{d \operatorname{Var}\left[Y^{(m)}\right]}{d m}}{\operatorname{Var}\left[Y^{(m)}\right]}+1 .
$$

Suppose now $Y$ is an aggregate sequence of packet counts resulting from the superposition of $M$ independent packet-traffic sources, not necessarily identical. Then $N(t)=N_{1}(t)+\cdots+N_{M}(t)$, where $N_{i}(t)$ denotes the number of packet arrivals in the interval $(0, t]$ from the $i^{\text {th }}$ traffic source. Assuming again stationarity, then:

$$
I D C(t)=\frac{\sum_{i=1}^{M} \operatorname{Var}\left[N_{i}(t)\right]}{\sum_{i=1}^{M} \lambda_{i} t}=\sum_{i=1}^{M}\left(\frac{I D C_{i}(t)}{\Lambda_{i}}\right)
$$

where $\lambda_{i}$ is the mean packet arrival rate from the $i^{t h}$ source, and $\Lambda_{i}=\frac{\sum_{j=1}^{M} \lambda_{j}}{\lambda_{i}}$. In addition, $\frac{\log (I D C(t))}{\log (t)}=$ $\frac{\log \left(\sum_{i=1}^{M} \operatorname{Var}\left[N_{i}(t)\right]\right)}{\log (t)}-\frac{\log \left(\sum_{i=1}^{M} \lambda_{i} t\right)}{\log (t)}$, and upon taking the derivative in respect to $\log (\mathrm{t})$ the Index of Variability for the aggregate traffic stream is computed to be:

$$
\begin{aligned}
H_{v}(\tau) & =0.5 \tau\left(\frac{\sum_{i=1}^{M} \frac{d \operatorname{Var}\left[N_{i}(\tau)\right]}{d \tau}}{\sum_{i=1}^{M} \operatorname{Var}\left[N_{i}(\tau)\right]}\right) \\
& =\frac{1}{2}\left\{1+\tau\left(\frac{\sum_{i=1}^{M} \frac{d\left(I D C_{i}(\tau)\right)}{d \tau}\left(\frac{1}{\Lambda_{i}}\right)}{\sum_{i=1}^{M}\left(\frac{I D C_{i}(\tau)}{\Lambda_{i}}\right)}\right)\right\} .
\end{aligned}
$$

As can be observed from (9), the variances or the indices of dispersion for counts of the $M$ independent point-processes completely characterize the variability function of the aggregate packet-count sequence $Y$. If $\lim _{\tau \rightarrow \infty} I D C(\tau)=\lim _{\tau \rightarrow \infty}\left(\sum_{i=1}^{M}\left(\frac{I D C_{i}(\tau)}{\Lambda_{i}}\right)\right)=c<\infty$, then obviously, $\lim _{\tau \rightarrow \infty} H_{v}(\tau)=0.5$. In the case that all $M$ underlying point processes of making up $Y$ are also identical, then (9) reduces to (6). If all $M$ underlying point processes are Poisson, then $\frac{d\left(I D C_{i}(\tau)\right)}{d \tau}=0$ for all $\tau$ and $i$ and hence $H_{v}(\tau)=0.5$ for all $\tau$.

\section{Analysis of Traffic Models in Terms of THE INDEX OF VARIABILITY}

In this section, we derive the Index of Variability functions for two traditional traffic models: two-state Markov
Modulated Poisson Process (MMPP) and renewal process with hyperexponential interarrival time distributions of order two (RPH2). Two-state MMPP models have become popular for modeling the superposition of packet voice streams [27].

The work in [28] shows that long-tail distributions can be approximated by hyperexponentional distributions. Thus, renewal processes with hyperexponential interarrival time distributions can be used for capturing the high variability of traffic over any range of (short or long) time scales. A major advantage of these models is their relative ease of analytically obtaining queueing performance predictions.

\section{A. Two-state MMPP}

Consider that the underlying point process of $Y$ is an MMPP with two-state Markov chain where the mean sojourn times in state 1 and 2 are $\alpha^{-1}$ and $\beta^{-1}$, respectively. When the chain is in state $i(i=1,2)$ the point process is Poisson with rate $\lambda_{i}$. Letting $\rho=$ $\alpha+\beta$ and $v=\lambda_{1} \beta+\lambda_{2} \alpha$, we have from [27] that $E[N(t)]=\frac{v t}{\rho}$ and $\operatorname{IDC}(t)=1+\rho A-A\left(\frac{1-e^{-\rho t}}{t}\right)$, where $A=\frac{2 \alpha \beta\left(\lambda_{1}-\lambda_{2}\right)^{2}}{\rho^{3} v}$. Clearly the $\lim _{t \rightarrow \infty} I D C(t)=1+\rho A$. Upon taking the derivative of $\operatorname{IDC}(\mathrm{t})$, the Index of Variability of $Y$ can be obtained:

$$
H_{v}(\tau)=0.5\left\{1+\frac{A\left[1-(1+\rho \tau) e^{-\rho \tau}\right]}{(1+\rho A) \tau-A\left(1-e^{-\rho \tau}\right)}\right\} .
$$

1) Numerical Example: Assume $\alpha^{-1}=\beta^{-1}=100$ seconds, $\lambda_{1}=4$ packets/second and $\lambda_{2}$ to vary from 1 to 1000 packets/second. Figure 2 shows the resulting index of variability curves as a function of time scale $(\tau)$ and state rates $\left(\lambda_{i}\right)$. Notice that when $\lambda_{2}=\lambda_{1}$, we have a pure Poisson process, and therefore zero variability; however, as the difference between $\lambda_{1}$ and $\lambda_{2}$ increases, so does the Index of Variability. From Figure 2 it can be observed that the Index of Variability increases with $\lambda_{2}$ up to its maximum value, and any further increase in $\lambda_{2}$ does not have any affect on variability. It can be also observed that the Index of Variability increases with $\tau$ up to its maximum value and then decays exponentially. In addition, notice for values of $\lambda_{2}$ further from $\lambda_{1}$, the packet-count process $Y$ has substantial variability over a wide range of time scales that spans about 200 seconds.

\section{B. $\mathrm{RPH} 2$}

Assume here that the underlying point process of $Y$ is a stationary renewal process with interarrival times hyperexponentially distributed. We call this model as the hyperexponential model. A hyperexponential distribution 

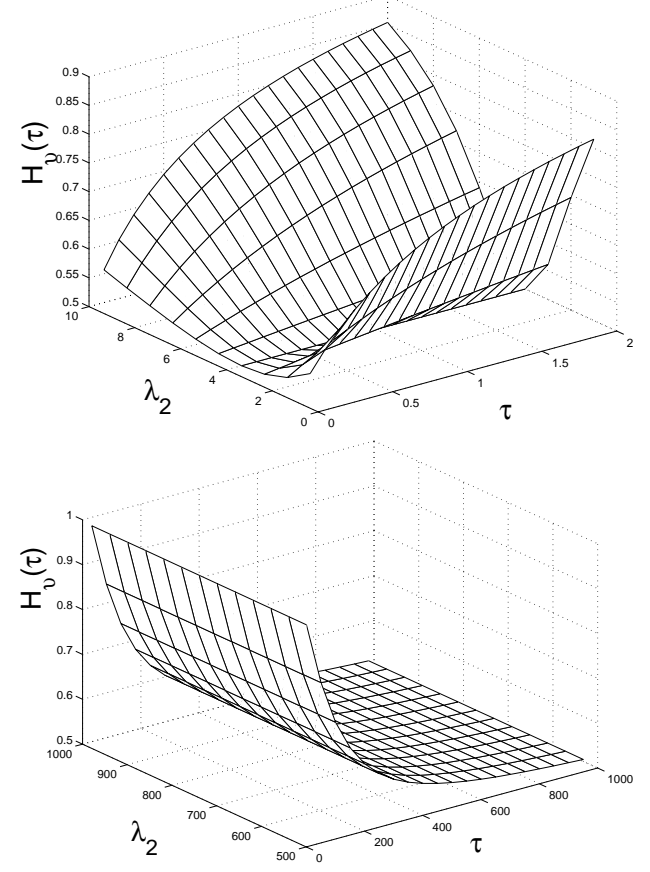

Fig. 2. Index of Variability for The Two-State MMPP: $\alpha^{-1}=\beta^{-1}=$ 100 Seconds, $\lambda_{1}=4$ Packets/Second.

of order $K,(=1,2,3, \ldots)$, is the weighted sum of $K$ exponential distributions:

$$
F_{K}(x)=\operatorname{Pr}[X \leq x]=\sum_{i=1}^{K} w_{i}\left(1-e^{-\alpha_{i} x}\right)
$$

where $w_{i}>0$ are the weights satisfying $\sum_{i=1}^{K} w_{i}=1$, and $\alpha_{i}>0$ are the rates of the exponential distributions [29]. It is shown in [30] that if $w_{i}=w^{i}$ and $\alpha_{i}=\frac{\mu}{\eta^{i}}$ for $0<w<$ $1, \eta>1$, and $\mu>0$, then the tail of the hyperexponential distribution gets longer and longer with $K$. The major advantages of the hyperexponential distributions over heavy-tailed distributions, such as Pareto are two-fold: their Laplace transform exists, therefore they can be utilized in analytic models, and they have finite variance for all $K$.

In this paper, we only consider the case of $K=2$. Letting $a=\alpha_{1}$ and $b=\alpha_{2}$, we get the pdf of the interarrival times to be:

$$
f_{2}(x)=w_{1} a e^{-a x}+w_{2} b e^{-b x} .
$$

The mean packet arrival rate is $\lambda=\frac{a b}{a w_{2}+b w_{1}}$, and the squared coefficient of variation of the interarrival times is $C^{2}(X)=2\left[\frac{a^{2} w_{2}+b^{2} w_{1}}{\left(a w_{2}+b w_{1}\right)^{2}}\right]-1$. Note that if $a=b$, then $\lambda=a=b$ and $C^{2}(X)=1$ for all the values of $w_{1}$ and $w_{2}$, and hence it is a Poisson process. In addition, $\lim _{w_{2} \rightarrow 0} C^{2}(X)=1$ and $\lim _{b \rightarrow 0} C^{2}(X)=\frac{2}{w_{2}}-1$. As shown in Fig. 3, for constant values of $a$ and $b, C^{2}(X)$ increases exponentially up to its maximum value and then

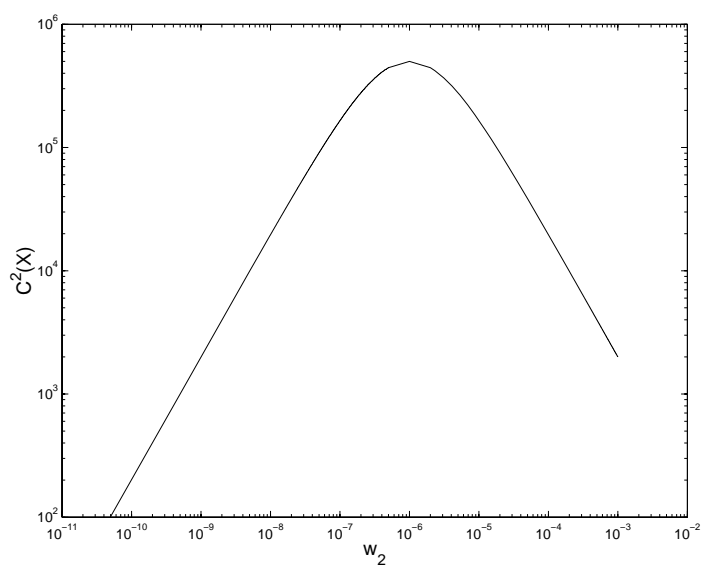

Fig. 3. Squared Coefficient of Variation of the Interarrival Time vs. $w_{2}$ for the Case of Hyperexponential Distribution of Order Two: $a=100$, and $b=0.0001$.

decreases to one very abruptly. This indicates that the hyperexponential distribution can be used to model the interarrival times distribution of highly bursty traffic.

From [31] we have that

$$
\operatorname{Var}[N(t)]=2 \lambda \int_{0}^{t} \Phi(u) d u+\lambda t-\lambda^{2} t^{2}
$$

where

$$
\Phi(t)=\mathcal{L}^{-1}\left[\Phi^{*}(s)\right]=\mathcal{L}^{-1}\left[\frac{f_{2}^{*}(s)}{s\left\{1-f_{2}^{*}(s)\right\}}\right] .
$$

Note that the symbol $\mathcal{L}^{-1}$ denotes the inverse Laplace transform and

$$
f_{2}^{*}(s)=\mathcal{L}\left[f_{2}(x)\right]=w_{1}\left(\frac{a}{s+a}\right)+w_{2}\left(\frac{b}{s+b}\right)
$$

is the Laplace transform of $f_{2}(x)$. Noting that:

$$
\begin{aligned}
\varphi(t) & =\mathcal{L}^{-1}\left[\frac{f_{2}^{*}(s)}{1-f_{2}^{*}(s)}\right] \\
& =\lambda-\frac{\left[\left(a w_{1}+b w_{2}\right)^{2}-\left(a^{2} w_{1}+b^{2} w_{2}\right)\right] e^{-\left[a w_{2}+b w_{1}\right] t}}{a w_{2}+b w_{1}}
\end{aligned}
$$

$\Phi(t)$ is obtained:

$$
\begin{aligned}
\Phi(t)= & \int_{0}^{t} \varphi(u) d u \\
= & \lambda t-\frac{\left[\left(a w_{1}+b w_{2}\right)^{2}-\left(a^{2} w_{1}+b^{2} w_{2}\right)\right]}{\left(a w_{2}+b w_{1}\right)^{2}} \\
& \left(1-e^{-\left[a w_{2}+b w_{1}\right] t}\right) .
\end{aligned}
$$

Performing the integration in (12) results:

$$
\operatorname{Var}[N(t)]=\frac{2 \lambda\left[\left(a w_{1}+b w_{2}\right)^{2}-\left(a^{2} w_{1}+b^{2} w_{2}\right)\right]}{\left(a w_{2}+b w_{1}\right)^{3}}
$$


TABLE I

Values of Mean Packet Rate $(\lambda)$ And Squared

COEFFICIENT OF VARIATION OF INTERARRIVAL TIMES $\left(C^{2}(X)\right)$

FOR THE NUMERICAL EXAMPLE OF THE CASE OF

HYPEREXPONENTIAL DISTRIBUTION OF ORDER TWO: $a=100$.

\begin{tabular}{|l|cc|cr|}
\hline & $\lambda$ & (packets/sec) & $C^{2}(X)$ & \\
$w_{2}$ & $b=0.01$ & $b=0.0001$ & $b=0.01$ & $b=0.0001$ \\
\hline \hline $10^{-3}$ & 9.1000 & 0.0999 & $1.6522 \times 10^{3}$ & $1.9950 \times 10^{3}$ \\
\hline $10^{-4}$ & 50.0000 & 0.9901 & $5.0000 \times 10^{3}$ & $1.9605 \times 10^{4}$ \\
\hline $10^{-5}$ & 90.9000 & 9.0909 & $1.6536 \times 10^{3}$ & $1.6529 \times 10^{5}$ \\
\hline $10^{-6}$ & 99.0000 & 50.0000 & 197.0202 & $5.0000 \times 10^{5}$ \\
\hline $10^{-7}$ & 99.9000 & 90.9091 & 20.9561 & $1.6529 \times 10^{5}$ \\
\hline $10^{-8}$ & 99.9900 & 99.0099 & 2.9992 & $1.9607 \times 10^{4}$ \\
\hline $10^{-9}$ & 99.9990 & 99.9001 & 1.2000 & $1.9970 \times 10^{3}$ \\
\hline $10^{-10}$ & 99.9999 & 99.9900 & 1.0200 & 200.9596 \\
\hline $10^{-11}$ & 100.0000 & 99.9990 & 1.0020 & 20.9996 \\
\hline $10^{-12}$ & 100.0000 & 99.9999 & 1.0002 & 2.9999 \\
\hline $10^{-13}$ & 100.0000 & 100.0000 & 1.0000 & 1.2001 \\
\hline
\end{tabular}

and hence

$$
\begin{aligned}
\frac{d}{d t}(\operatorname{Var}[N(t)])= & \frac{2 \lambda\left[\left(a w_{1}+b w_{2}\right)^{2}-\left(a^{2} w_{1}+b^{2} w_{2}\right)\right]}{\left(a w_{2}+b w_{1}\right)^{2}} \\
& e^{-\left[a w_{2}+b w_{1}\right] t}+\lambda c^{2}(X),
\end{aligned}
$$

and

$$
\begin{aligned}
I D C(t)= & \frac{2\left[\left(a w_{1}+b w_{2}\right)^{2}-\left(a^{2} w_{1}+b^{2} w_{2}\right)\right]}{\left(a w_{2}+b w_{1}\right)^{3}} \\
& \left(\frac{1-e^{-\left[a w_{2}+b w_{1}\right] t}}{t}\right)+C^{2}(X) .
\end{aligned}
$$

Observe that $\lim _{t \rightarrow \infty} I D C(t)=C^{2}(X)$, and if $a=b$ then $\left[\left(a w_{1}+b w_{2}\right)^{2}-\left(a^{2} w_{1}+b^{2} w_{2}\right)\right]=0$ and $C^{2}(X)=1$ making $\operatorname{Var}[N(t)]=\lambda t$ and $I D C(t)=1$, i.e., we get a Poisson process. (16) or (18) can then be used in (5) or (6) to obtain the index of variability. It is obvious to see that $\lim _{\tau \rightarrow \infty} H_{v}(\tau)=0.5$.

Deriving the symbolic expression of $\operatorname{Var}[N(t)]$ for $K>2$ is a difficult problem, mainly due to the difficulty in deriving $\phi(t)$, i.e., performing the following inverse Laplace transform:

$$
\mathcal{L}^{-1}\left[\frac{f_{K}^{*}(s)}{1-f_{K}^{*}(s)}\right]
$$

where $f_{K}^{*}(s)$ is the Laplace transform of the the $K$-order hyperexponential pdf of the interarrival times. However, it becomes trivial when the model parameters (e.g., $w_{i}$ and $\alpha_{i}$ ) are set to numerical values.

1) Numerical Example: Let $a=100$. Table I lists the values of the mean packet rate $(\lambda)$ and the squared coefficient of variation of the interarrival times $\left(C^{2}(X)\right)$ for $b=0.01$ and $b=0.0001$ for different values of $w_{2}$. Note that $w_{1}+w_{2}=1$. Interestingly, the maximum value of $C^{2}(X)$ occurs when $\lambda=\frac{a}{2}$. Also, Fig. 4 indicates that at this value of $\lambda$, the process attains the widest range of time scales of high variability, and in this range the index of variability reaches its maximum value (curve (i), $\left.\operatorname{maximum} H_{v}=0.9988\right)$. Observe that this widest range

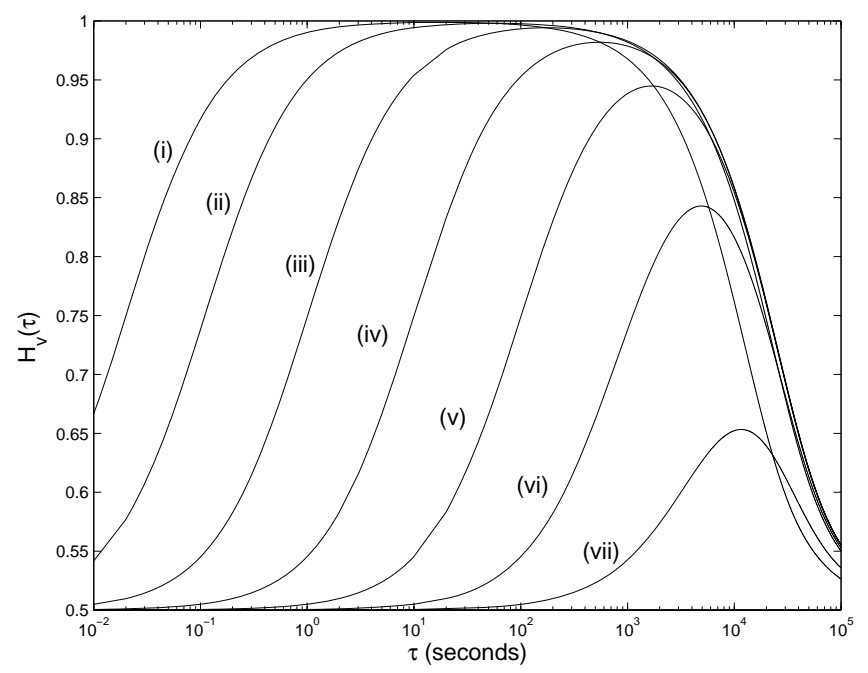

Fig. 4. Index of Variability vs. Time Scale for the Case of Hyperexponential Distribution of Order Two: $a=100, b=0.0001$, $w_{2}=$ (i) $10^{-6}$ (ii) $10^{-7}$ (iii) $10^{-8}$ (iv) $10^{-9}$ (v) $10^{-10}$ (vi) $10^{-11}$ (vii) $10^{-12}$.

of time scales of high variability most likely covers all time scales that impact network performance evaluation [7]. In this example and for $\lambda=\frac{a}{2}$ packets/s, the range of time scales for which the packet-count sequence $Y$ exhibits high variability spans 7 order of magnitude.

In addition, Figure 4 shows that the maximum value of variability as well as the range of time scales of substantial variability become smaller as $\lambda \rightarrow a$. Let

$\tau_{o n}=$ inf $\{$ range of time scales of substantial variability $\}$,

and

$\tau_{o f f}=\sup \{$ range of time scales of substantial variability $\}$.

As can be observed from these curves, $\tau_{\text {on }}$ gets bigger as $\lambda$ approaches $a$. Although it is not completely shown in Figure 4, it is not difficult to see that $\tau_{\text {off }}$ becomes smaller as $\lambda \rightarrow b$. Notice that for all $\tau \ni\left[\tau_{o n}, \tau_{o f f}\right]$ the process behaves like Poisson.

Figure 5 depicts 3D Index of Variability curves generated using the hyperexponential model with $K=2$. Clearly, both Figures 4 and 5 demonstrate that the hyperexponential model can yield a variety of Index of Variability curves. Hence, hyperexponential models can be used to model a wide range of network traffic types. Although hyperexponential models of order two (i.e., $K=2$ ) are capable of generating a variety of Index of Variability curves, capturing the characteristics of 

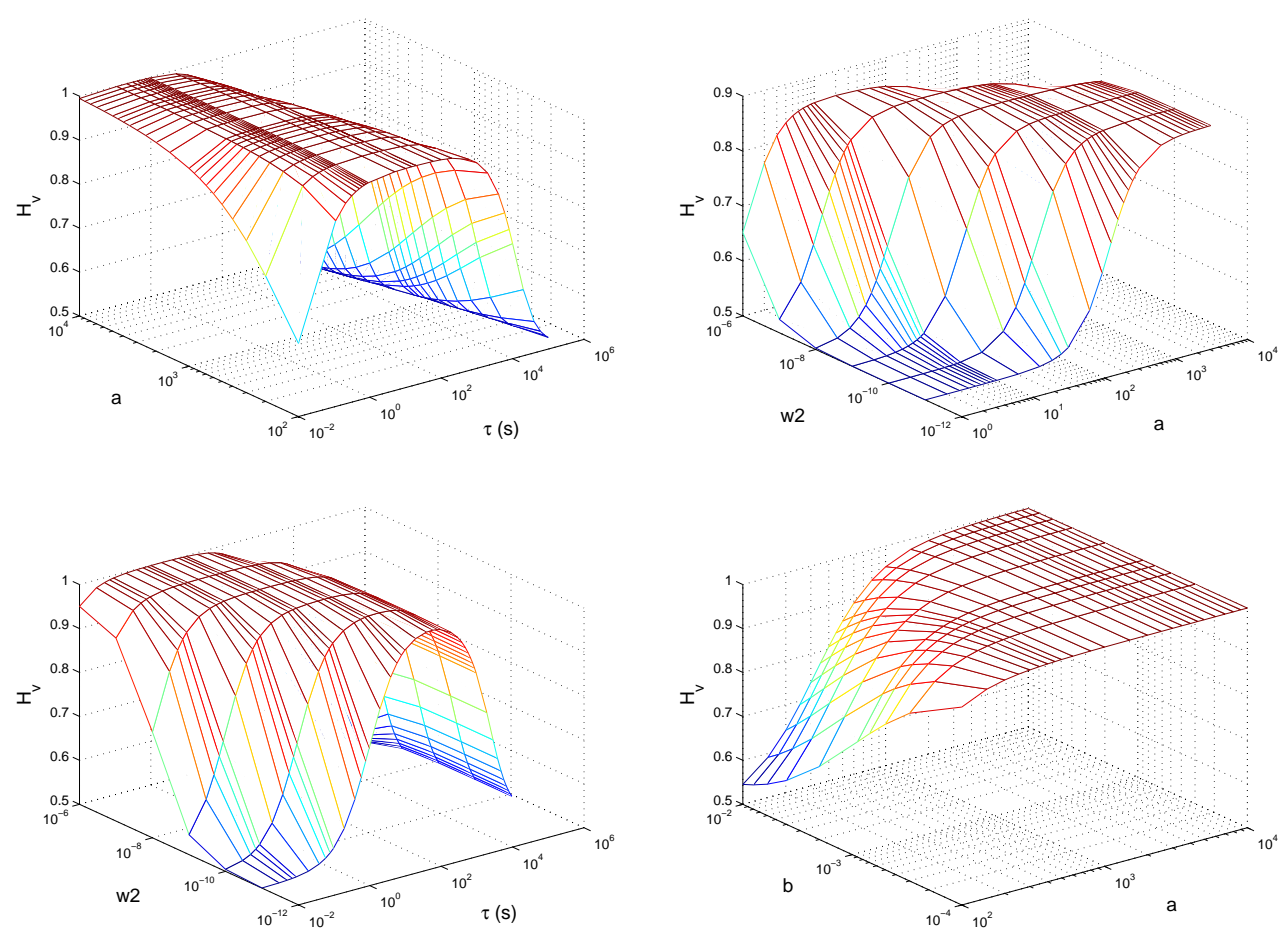

Fig. 5. Illustrations of 3D Index of Variability curves generated using the hyperexponential model with the following model parameter values: (top left) $b=0.0001, w_{2}=10^{-6}$ (top right) $\tau=1000, b=0.001$ (bottom left) $a=1000, b=0.0001$ (bottom right) $\tau=1, w_{2}=10^{-7}$.

traffic with multimodal Index of Variability curves would require using higher order $(K>2)$ hyperexponential models.

\section{ESTIMATING $H_{v}(\tau)$ FROM TRAFFIC TRACES}

The estimation of the Index of Variability curve from a given traffic trace requires the estimation of the first derivative of $\operatorname{Var}[N(\tau)]$ from discrete samples $\left(\operatorname{Var}\left[N\left(\tau_{i}\right)\right], i=1, \cdots, n\right)$. To do this, we must first find an analytic function that best fits the discrete variance data. This in turn requires the use of an interpolation method such as polynomial-based interpolation, cubic spline and smoothing spline [35]-[39].

Since we use the sample variances as the estimates of $\operatorname{Var}\left[N\left(\tau_{i}\right)\right], i=1, \cdots, n$, we consider these estimates of the variances to be noisy samples. The smoothing spline interpolation methods are known to have optimal properties for estimating continuous functions and their derivatives from a finite number of noisy samples [36], [38], [39]. Note that nonsmoothing interpolation methods such as cubic spline have the characteristic that the estimated curve passes through all the given points. Hence, in case of noisy data, nonsmoothing interpolation methods yield rough curves, and therefore erroneously high first derivatives.

\section{A. Smoothing Spline Interpolation Method}

For a given data series $\left(x_{i}, y_{i}\right), i=1,2, \cdots, n$, the smooth function $f(x)$ is the solution of the minimization problem

$$
\frac{1}{n} \sum_{i=1}^{n}\left(y_{i}-f\left(x_{i}\right)\right)^{2}+\xi \int_{x_{1}}^{x_{n}}\left(f^{(k)}\right)^{2} d u
$$

where $\xi$ is the smoothing parameter and $f^{(k)}$ is the $k^{\text {th }}$ derivative of $f$. If $k=2$, then $f$ is a cubic smoothing spline.

The first term in (19) is the residual sum of squares, an indicator of the goodness-of-fit of the spline curve to the data. In other words, it measures the degree of fidelity of the smoothing spline function to the data. The second term measures the roughness of the resulting smoothing spline curve. The roughness of a function can be characterized by its curvature. For example, if a function is a straight line, then its second derivative (and therefore, roughness) is zero, that is, the second term is a penalty term measuring how close the function is to a straight line.

The smoothing parameter $\xi$ plays an important role. It weights two aspects: smoothness and fit. Large values of $\xi$ give a smoother curve, while small values of $\xi$ result in a closer fit. 


\section{B. Steps for Estimating $H_{v}(\tau)$ from Traffic Traces}

We now present a practical method for estimating the Index of Variability from traffic traces. Assuming that a given traffic trace is a realization of a second-order ergodic point process whose variance curve is continuous and differentiable. We can estimate $H_{v}(\tau)$ of the process as follows:

- Using the Aggregated Variance method [11] estimate the variance-time sequence: $\widehat{\operatorname{Var}}\left[N\left(\tau_{i}\right)\right], i=$ $1, \cdots, n$.

- Using an appropriate smoothing spline implementation estimate the smoothing spline $\widehat{\operatorname{Var}}[N(\tau)]$ from $\widehat{\operatorname{Var}}\left[N\left(\tau_{i}\right)\right], i=1, \cdots, n$.

- Using (5) estimate the Index of Variability $\widehat{H_{v}}(\tau)$.

Prior to experimentation, the accuracy of this process was validated by estimating and matching the Index of Variability curves shown in Figure 4 from synthetically generated data using the hyperexponential traffic model.

\section{Experimental Results}

This section presents experimental results that demonstrate the robustness of our method for estimating the Index of Variability functions from traffic traces, as described in the previous section.

Using the steps outlined in the previous section, we estimated the Index of Variability curve $\left(H_{v}(\tau)\right)$ from 12 NLANR network traffic long traces [34]. The dates at which each trace was collected and their durations are listed in Table II. For more information about these traffic traces, see [34]. Figures 6 and 7 show the estimated Index of Variability curves from the 12 long packet traces.

We used Matlab's spline toolbox to estimate all the smoothing splines. Its smoothing spline implementation is based on Reinsch's approach [36], [37]. Based on the input data, the algorithm computes the optimal smoothing parameter $\xi$ such that the penalized residual sum of squares is less than a tolerance value $\varepsilon>0$. In all cases we used the default value of $k(=2)$ and $\varepsilon=0.0001$.

\section{DISCUSSION}

As expected, the results displayed in Figures 6 and 7 show that the variability of real network traffic varies with time scales. An interesting observation is that the Index of Variability curves derived from the Auckland traffic traces exhibit similar monomodal behavior, while the Bell-Lab Index of Variability curve is multimodal. Hence, hyperexponential models of order two can be used to well approximate the Auckland traffic processes (see Figures 4 and 5), but they are not appropriate to be used to capture the characteristics of the Bell Lab traffic.
We believe that the characteristics exhibited by the Bell Lab traffic can be captured either by hyperexponential models of order higher than two or by mixprocess models, that is the superposition of heterogeneous traffic processes. As an illustration, consider that an aggregated network traffic (packet) process is the result of the superposition of 10 renewal processes with hyperexponential interarrival time distribution of order two (RPH2), 20 two-state Markov Modulated Poisson processes (MMPP), 16 packetized voice streams, and 40 packet streams generated by ON/OFF traffic sources whose ON and OFF periods are both exponentially distributed $^{4}$. The resulting Index of Variability curve for this mixprocess traffic model is shown in Figure 8. Note that the dash-line curve is the $H_{v}$ curve for the case of aggregating only 10 identical RPH2 processes. Comparing the Index of Variability curves of Figures 6 and 8 , it can be inferred that the Bell-Lab traffic can be well modeled using such a mixprocess traffic model.

Evidently, the Index of Variabilty is a valuable tool that can provide new insights and understanding of the dynamics of network traffic. $H_{v}$ curves can be used to identify the characteristics of networks or applications that generate the various types of traffic patterns. An $H_{v}$ curve can be regarded as a "signature" of a particular network or application. To further understand the difference between the Auckland and Bell-Lab Index of Variability curves, these two networks must be compared in terms of their architecture, protocols employed at each layer, physical links, applications, and other network specific characteristics.

\section{CONCLUSION}

Knowledge of the traffic characteristics on multiple time scales can help to improve the efficiency of traffic control mechanisms. In addition, the design and provision of quality-of-service-guarantees over the Internet requires the understanding of traffic characteristics, such as variability. This paper has presented an alternative measure of traffic burstiness called the Index of Variability $\left(H_{v}(\tau)\right)$, that fully and accurately describes the degree of variability of a typical network traffic process at each time scale and is analytically tractable for many traffic models. Analytical derivations of $H_{v}(\tau)$ were given for two conventional traffic models. Several generated 2D and 3D Index of Variability curves predicate that the Index of Variability is a mathematically rigorous measure which can be used to fully characterize the complexities of the network traffic variability over all time scales.

\footnotetext{
${ }^{4}$ See [33] for more details.
} 

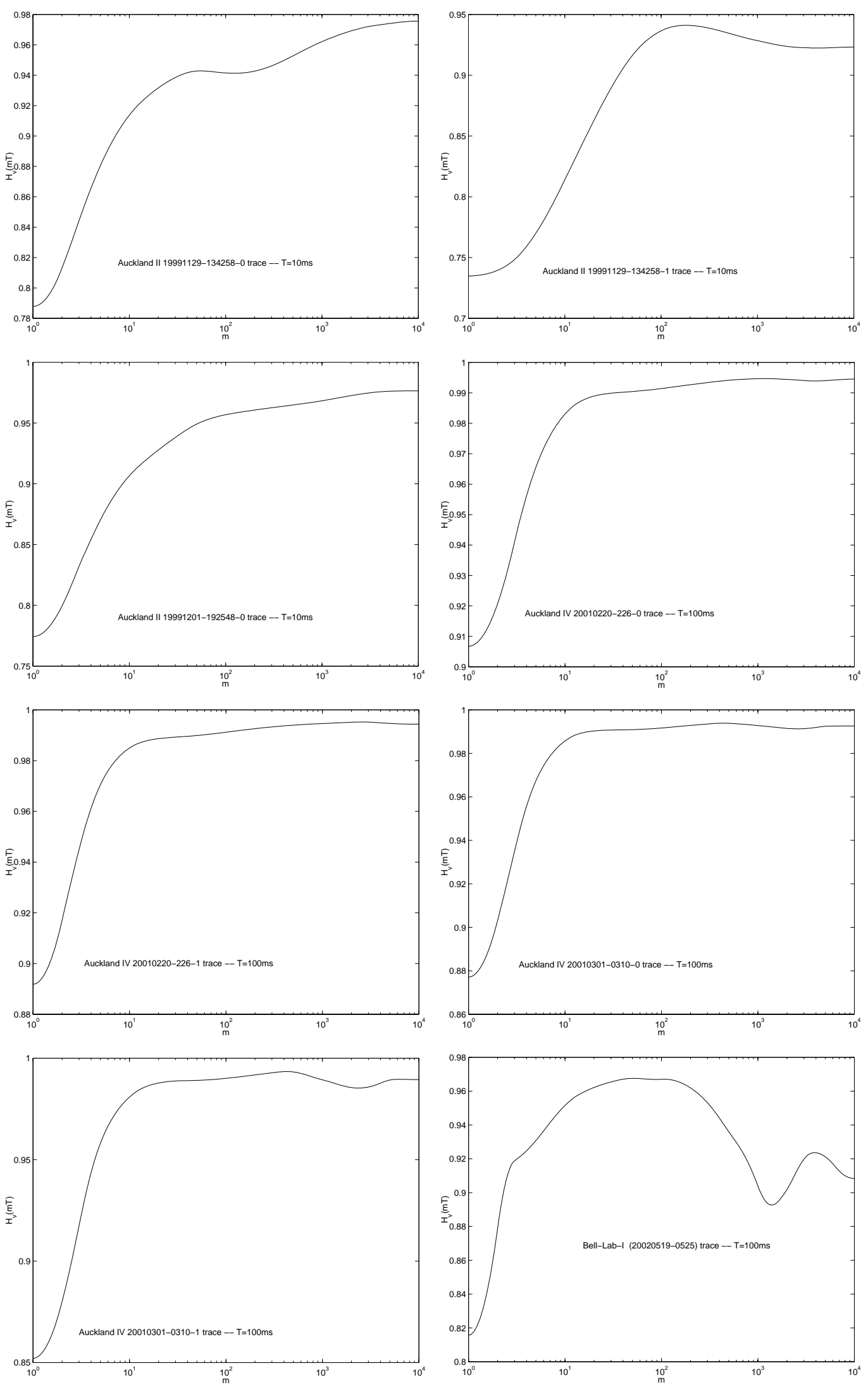

Fig. 6. Estimated Index of Variability Curves for Auckland II (top first three), Aucland IV (next four), and Bell-Lab-I (last) traces 
TABLE II

NLANR NETWORK TRAFFIC TRACES

\begin{tabular}{|l|c|c|c|}
\hline Trace & Data Set & Date Collected & $\begin{array}{c}\text { Duration } \\
\text { (Days:Hours:Minutes) }\end{array}$ \\
\hline \hline $19991129-134258-0$ & Auckland-II & November 29, 1999 & $1: 14: 29$ \\
\hline $19991129-134258-1$ & Auckland-II & November 29, 1999 & $1: 14: 29$ \\
\hline $19991201-192548-0$ & Auckland-II & December 1, 1999 & $1: 0: 2$ \\
\hline $20010220-226-0$ & Auckland-IV & February 20, 2001 & $6: 4: 58$ \\
\hline $20010220-226-1$ & Auckland-IV & February 20, 2001 & $6: 4: 58$ \\
\hline $20010301-0310-0$ & Auckland-IV & March 1, 2001 & $9: 14: 49$ \\
\hline $20010301-0310-1$ & Auckland-IV & March 1, 2001 & $9: 14: 49$ \\
\hline $20010609-0613-0$ & Auckland-VI & June 9, 2001 & $4: 6: 0$ \\
\hline $20010609-0613-1$ & Auckland-VI & June 9, 2001 & $4: 6: 0$ \\
\hline $20010609-0613-e 0$ & Auckland-VI & June 9, 2001 & $4: 6: 0$ \\
\hline $20010609-0613-e 1$ & Auckland-VI & June 9, 2001 & $4: 6: 0$ \\
\hline $20020519-525$ & Bell-Lab-I & May 19, 2002 & $7: 0: 0$ \\
\hline
\end{tabular}
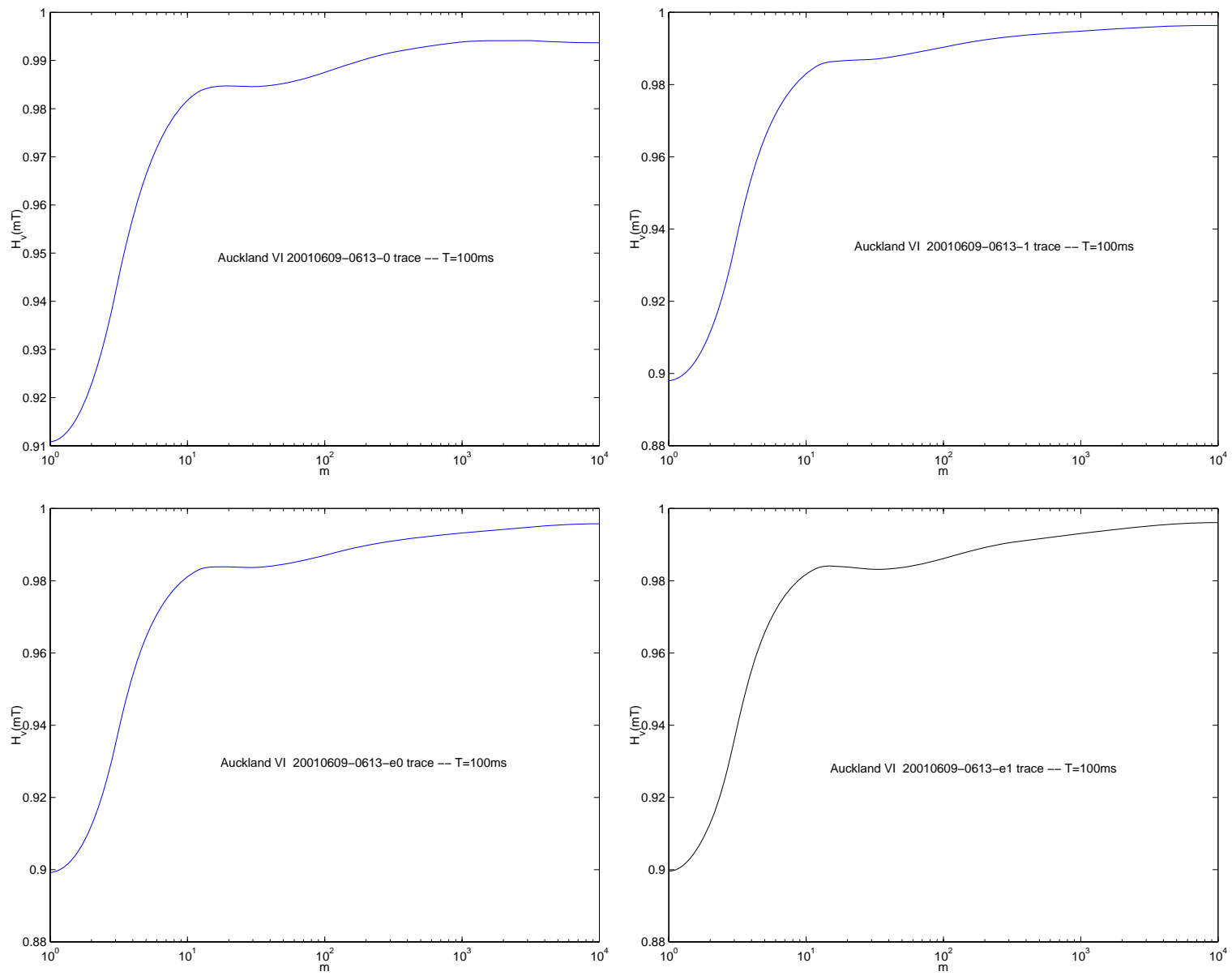

Fig. 7. Estimated Index of Variability Curves for Auckland VI traces 


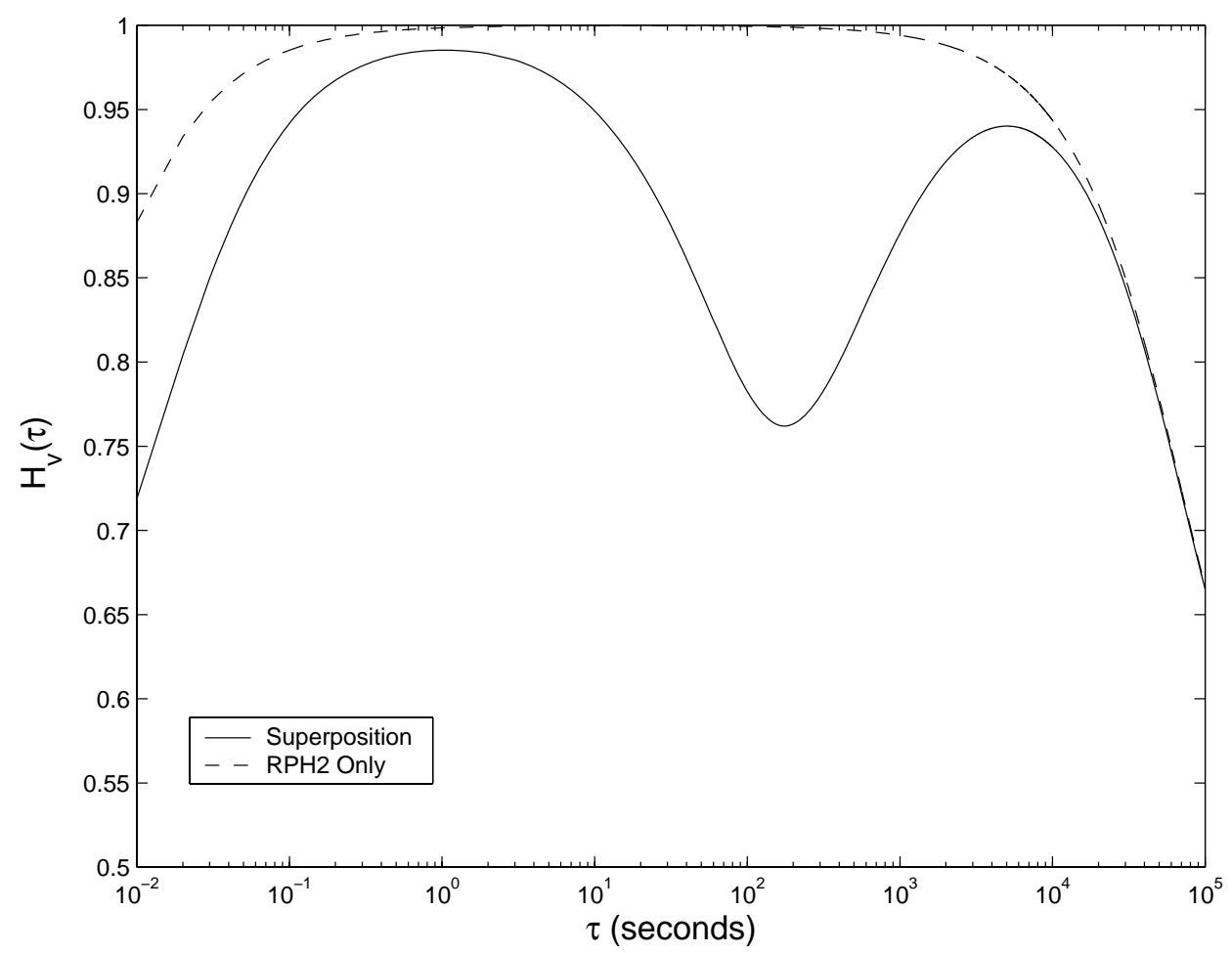

Fig. 8. Analytically derived Index of Variability curve for the case of mixprocess traffic model example.

This paper has also presented a practical method for estimating the Index of Variability curves from traffic traces. Experimental results validated the robustness of the method when applied to the estimation of the Index of Variability curves from 12 NLANR network traffic long traces. In summary, the Index of Variability offers the potential to gain insights into the dynamics of network traffic that existing tools do not offer. Future work involves developing methods of fitting analytically obtained Index of Variability functions to empirically obtained Index of Variability curves. To accomplish this, nonlinear optimization techniques will be utilized. In addition, studies to find relations that associate $H_{v}(\tau)$ with queuing performance metrics, such as packet loss rate and delay, will be performed.

\section{REFERENCES}

[1] G.Y. Lazarou, X. Xiangdong and V.S. Frost, "Internet Traffic Modeling Using the Index of Variability," in Proc. IASTED International Conference on Modeling and Simulation, 2003, pp. 31-37.

[2] D. Duffy, A. McIntosh, M. Rosenstein and D. Wilson, "Statistical Analysis of CCSN/SS7 Traffic Data from Working CCS Subnetworks," IEEE JSAC, vol. 12, no. 3, pp. 544-551, April 1994.

[3] H.J. Fowler and W.E. Leland, "Local Area Network Traffic Characteristics, with Implication for Network Congestion Management," IEEE JSAC, vol. 9, no. 7, pp.1139-1149, September 1991.
[4] W. Leland, M. Taqqu, W. Willinger and D. Wilson, "On the Self-Similar Nature of Ethernet Traffic (Extended Version)," IEEE/ACM Trans. on Networking, vol.2, no.1, pp. 1-15, February. 1994.

[5] V. Paxson and S. Floyd, "Wide-Area Traffic: The Failure of Poisson Modeling," IEEE/ACM Trans. on Networking, vol. 3, no. 3, pp. 226-244, June 1995.

[6] A. Erramilli, O. Narayan and W. Willinger, "Experimental Queueing Analysis with Long-Range Dependent Packet Traffic,' IEEE/ACM Trans. on Networking, vol. 4, no. 2, pp. 209-223, April 1996.

[7] M. Grossgluser and J.-C. Bolot, "On the Relevance of LongRange Dependence in Network Traffic," IEEE/ACM Trans. on Networking, vol. 7, no. 5, pp. 629-640, October 1999.

[8] K. R. Krishnan, A. L. Neidhardt and A. Erramilli, "Scaling Analysis in Traffic Management of Self-Similar Processes," in Proc. 15th ITC, 1997, pp. 1087-1096.

[9] A.L. Neidhardt and J.L. Wang, "The Concept of Relevant Time Scales and Its Application to Queueing Analysis of Self-Similar Traffic (or Is Hurst Naughty or Nice?)," in Proc. ACM SIGMETRICS, 1998), pp. 222-232.

[10] K. Park and W. Willinger, Editors, Self-Similar Network Traffic And Performance Evaluation, New York: John Wiley \& Sons, 2000.

[11] J. Beran, Statistics for Long-Memory Processes. Monographs on Statistics and Applied Probability, New York: Chapman and Hall, 1994.

[12] M. S. Taqqu, V. Teverovsky and W. Willinger, "Estimators for Long-range Dependence: An Emperical Study," Fractals, vol. 3 , no. 4, pp. 785-798, 1995.

[13] D. Veitch and P. Abry, "A Wavelet Based Joint Estimator for the Parameters of LRD," Special issue on Multiscale Statistical Signal Analysis and its Applications, IEEE Trans. Information Theory, vol. 45, no. 3, April 1999.

[14] A. Baiocchi, N. Blefari Melazzi, M. Listanti, A. Roveri and R. 
Winkler, "Modeling Isssues on an ATM Multiplexer Within a Bursty Traffic Environment," in Proc. IEEE INFOCOM, 1991, pp. 83-91.

[15] I. Norros, "A storage model with self-similar input," Queueing Systems, vol. 16, pp. 387-396, 1994.

[16] W. E. Leland and D. V. Wilson, "High Time-Resolution Measurement and Analysis of LAN Traffic: Implication for LAN Interconnection," in Proc. IEEE INFOCOM, 1991, pp. 13601366.

[17] K. R. Krishnan, "A New Class of Performance Results for a Fractional Brownian Traffic Model," Queueing Systems, vol. 22, pp. 277-285, 1996.

[18] Q. Li and D. Mills, "Investigating the Scaling Behavior, Crossover and Anti-persistence of Internet Packet Delay Dynamics," in Proc. IEEE Globecom, 1999, pp. 1843-1852.

[19] V.J. Ribeiro, R.H. Reidi and R.G. Baraniuk, "Wavelets and Multifractals for Network Traffic Modeling and Inference," in Proc. IEEE ICASSP, 2001, pp. 3429-3432.

[20] K. Kant, "On aggregate Traffic Generation with Multifractal Properties," in Proc. IEEE Globecom, 1999, pp.801-804.

[21] R.H. Riedi, M.S. Crouse, V.J. Ribeiro and R.G. Baraniuk, "A Multifractal Wavelet Model with Application to Network Traffic," IEEE Trans. on Information Theory, vol. 45, no. 3, pp. 992-1018, April 1999.

[22] J. Levy Vehel and B. Sikdar, "A Multiplicative Multifractal for TCP Traffic," in Proc. of 6th IEEE Symposium on Computers and Communications, 2001, pp. 714-719.

[23] A.C. Gilbert, W. Willinger and A. Feldmann, "Scaling Analysis of Conservative Cascades, with Applications to Network Traffic," IEEE Trans. on Information Theory, vol. 45, no. 3, pp. 971-991, April 1999.

[24] M.S. Taqque, Vadim Teverovsky and W. Willinger, "Is Network Traffic Self-Similar or Multifractal?” Fractals, vol. 5, pp. 63-73, 1997.

[25] A. Horvath and M. Telek, "A Markovian Point Process Exhibiting Multifractal Behaviour and its Application to Traffic Modeling," in Proc. 4th International Conference Matrix Analytic Methods in Stochastic Models, 2002.

[26] W. Willinger, V. Paxson, R.H. Riedi and M. Taqqu, "LongRange Dependence and Data Network Traffic," Long-range Dependence: Theory and Applications, P. Doukhan, G. Oppenheim, M.S. Taqqu, Eds., Birkhauser, 2002.

[27] H. Heffes and D.M. Lucantoni, "A Markov Modulated Characterization of Packetized Voice and Data Traffic and Related Statistical Multiplexer Performance," IEEE JSAC, vol. SAC-4, no. 6, pp. 856-868, September 1986.

[28] A. Feldman and W. Whitt, "Fitting Mixtures of Exponentials to Long-Tail Distributions to Analyze Network Performance Models," Performance Evaluation, vol. 31, no. 8, pp. 963-976, August 1998.

[29] R. Gusella, "Characterizing the Variability of Arrival Processes with Indexes of Dispersion”, IEEE JSAC, vol. 9, no. 2, pp. 203211, February 1991.

[30] M. Greiner, M. Jobmann and L. Lipsky, "The Importance of Power-Tail Distributions for Telecommunications Traffic Modeling," Institute für Informatik, Technische Universität München, München, Germany, Technical Report, 1995.

[31] D.R. Cox, Renewal Theory, London: J. Wiley \& Sons, 1962.

[32] P.M. Fiorini, Modeling Telecommunication Systems with SelfSimilar Data Traffic, Ph.D. Dissertation, University of Connecticut, USA, September 9, 1998.

[33] G. Y. Lazarou, On the Variability of Internet Traffic, Ph.D. Dissertation, Electrical Engineering and Computer Science, University of Kansas, 2000.

[34] J. Micheel, NLANR PMA: Special Traces Archive, [Online]. Available: http://pma.nlanr.net/Traces/long/
[35] R.L. Burden and J.D. Faires, Numerical Analysis $7^{\text {th }}$ ed., Brooks/Cole, Thomson Learning, Inc., 2001.

[36] C.M. Reinsch, "Smoothing by Spline Functions," Numerische Mathematik, vol. 10, pp. 177-183, 1967.

[37] R.L. Eubank, Spline Smoothing and Nonparametric Regression, New York:Marcel Dekker, 1988.

[38] J. Fox, Nonparametric Simple Regression: Smoothing Scatterplots, Californian:Sage Publications, Thousand Oaks, 2000.

[39] B. Shahraray and D.J. Anderson, "Optimal Estimation of Contour Properties by Cross-Validated Regularization," IEEE Trans. on Pattern Analysis and Machine Intelligense, vol. 11, no. 6, pp. 600-610, June 1989.

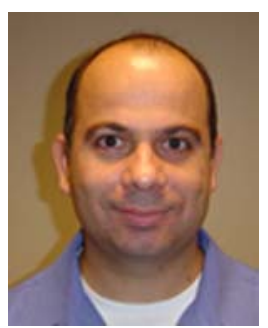

Georgios Y. Lazarou is currently an Assistant Professor in the Electrical and Computer Engineering Department at Mississippi State University. He is a founding member of the Telecommunication and Information Technology Laboratory (TITL) at Mississippi State University. Currently, his is member of the Intelligent Electronic Systems (IES) program at CAVS. His current research interests lie in the area of next generation smart networking technologies, wireless networking for intelligent transportation systems, and energy efficient protocols for wireless sensor networks. He has been involved in research projects concerning the design of adaptive and reconfigurable networks, modeling and evaluating the performance of high-speed wide-area asynchronous transfer mode (ATM) networks and Internet, and characterization of network traffic. Results from his work have been published in numerous journal and conference articles, and in technical reports.

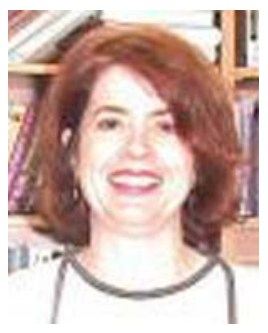

Julie Baca is currently a Research Assistant Professor at the Center for Advanced Vehicular Systems at the Mississippi State University. Prior to this, Dr. Baca was a member of the computer science research group of the Information Technology Laboratory at U.S. Army Corps of Engineers. Currently, Dr. Baca directs the mobile computing usability and the in-vehicle dialogue interface projects with an emphasis on voice over IP. She has over a decade of experience in designing complex software system interfaces for voice and multimedia applications over the Internet. Her research interests include mobile computing, wireless networking, operating systems, and user interface design in mobile environments. Dr. Baca received her Ph.D. degree from Mississippi State University in 1998, and is a member of ACM. 


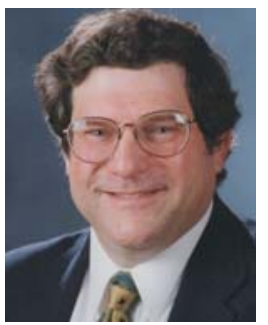

Victor S. Frost is currently the Dan F. Servey Distinguished Professor of Electrical Engineering and Computer Science and Director of the University of Kansas Telecommunications and Information Technology Center (ITTC). $\mathrm{He}$ is a Fellow of the IEEE and received a Presidential Young Investigator Award from the National Science Foundation in 1984. His current research interest is in the areas of internet quality of service, traffic management, and integrated broadband communication networks. He has been involved in research on several national scale high speed wide area testbeds. Government agencies, including, NSF, DARPA, Rome Labs, and NASA have sponsored his research. Professor Frost has been involved in research for numerous corporations, including Harris, Sprint, NCR, BNR, Telesat Canada, AT\&T, McDonnell Douglas, DEC, and COMDISCO Systems. He has published over 100 journal articles and conference papers. Dr. Frost received the BS, MS, and Ph.D. degrees from the University of Kansas, Lawrence in 1977, 1978, and 1982, respectively. In 1982 he joined the faculty of the University of Kansas

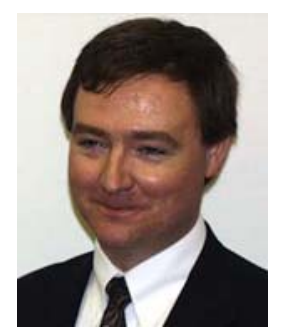

Joseph B. Evans is the Deane E. Ackers Distinguished Professor of Electrical Engineering \& Computer Science and Director of Research Information Technology at the University of Kansas. He recently served as a Program Director in the Division of Computer \& Network Systems in the Directorate for Computer \& Information Science \& Engineering at the National Science Foundation. His research interests include mobile and wireless networking, pervasive computing systems, high speed networks, and adaptive computing systems. He has been involved in major national high performance networking testbeds and broadband wireless mobile networking efforts, and has published over 100 journal and conference works. Dr. Evans received his $\mathrm{PhD}$ degree from Princeton University in 1989, is a senior member of the IEEE, and a member of the ACM. 